\title{
ESTIMULANDO MENINAS DO MARANHÃO PARA AS CARREIRAS DE STEM
}

\begin{abstract}
Kátia Simone Teixeira da Silva de La Salles - katia.salles@ufma.br*
Wendell Ferreira de La Salles - wendell.salles@ufma.br*

Departamento de Tecnologia Química
\end{abstract}

Paulo Rogério de Almeida Ribeiro - paulo.ribeiro@ecp.ufma.br*

Coordenação de Engenharia da Computação

Janyeid Karla Castro Sousa - janyeid.castro@ufma.br*

Coordenação de Ciência e Tecnologia

Maria de Fatima Santos - mf.santos@ufma.br**

Departamento de Engenharia de Eletricidade

Carlos de Salles Soares Neto - csalles@ deinf.ufma.br*

Luís Jorge Enrique Rivero Cabrejos - luis.rivero@ufma.br*

Departamento de Ciência da Computação

Valeska Martins de Souza - valeska.martins@ufma.br*

Departamento de Matemática

*Universidade Federal do Maranhão

Av. dos Portugueses, 1966, Bacanga

CEP 65080-805 - São Luís - Maranhão

Resumo: Visando desmistificar a área de Ciência, Tecnologia, Engenharia e Matemática (STEM - Science, Technology, Engineering and Mathematics), assim como despertar o interesse de meninas a seguir essa carreira, um grupo de professores das áreas de ciência, tecnologia, engenharia e matemática da Universidade Federal do Maranhão propôs o projeto "Sarmininal Cientistas: Estimulando Meninas do Maranhão para as Carreiras de Exatas e Tecnologia". O projeto adotou como metodologia uma abordagem dinâmica - isto é atividades lúdicas e ativas de aprendizagem - para promover o encantamento, o estímulo e a curiosidade das alunas de escolas públicas de São Luís do Maranhão para as áreas de STEM. Das diversas ações realizadas, podemos citar as atividades na área de robótica; oficinas de desenvolvimento do pensamento computacional e de introdução a SCILAB e MATLAB; mostras de filmes, além de palestras de orientação profissional. Todas as ações foram planejadas no sentido de proporcionar às alunas a aproximação com uma área muitas vezes minorada por grande parte dos estudantes, principalmente pelo público feminino. Ao longo das ações, foi possível observar vários impactos positivos, de modo que pudemos concluir que as atividades ajudaram as alunas a perceberem a área de STEM de uma forma diferente, correlacionando os assuntos abordados com as disciplinas de matemática, física, química e computação. 
Palavras-chave: Meninas. STEM. Escola Pública. Sarminina Cientistas.

\section{INTRODUÇÃO}

De acordo com um relatório da UNESCO, as adolescentes não buscam as ciências exatas na mesma proporção que os meninos (NORTE, 2018). Diversos são os motivos para ocorrência desse fato: desigualdade de gênero, educação sexista, estereótipos de gênero no ambiente escolar, entre outros.

O desinteresse das meninas por ciências e estudos técnicos é uma questão social; os modelos existentes constroem, desde a infância, um padrão profissão versus gênero (UNESCO, 2003). Esse comportamento tem repercussões no ensino superior, onde há um desequilíbrio de gênero nos cursos das áreas de Ciências, Tecnologia, Engenharia e Matemática (STEM, sigla em inglês), (SIRIMANNE, 2019).

Buscar meios para maior representatividade das mulheres nas áreas de STEM e a permanência delas nessas áreas são desafios a serem enfrentados por todos os países (BOLZANI, 2017).

Na busca por desmistificar a área de STEM e despertar o interesse de meninas a seguir carreira nesta, um grupo de professores das áreas de ciência, tecnologia, engenharia e matemática propôs o projeto "Sarminina Cientistas: Estimulando Meninas do Maranhão para as Carreiras de Exatas e Tecnologia", que conta com o apoio do Conselho Nacional de Desenvolvimento Científico e Tecnológico (CNPq). Esse projeto reafirma o vínculo entre a universidade e escolas, possibilitando à comunidade acadêmica atuar em um projeto multidisciplinar, articulando ensino, pesquisa e extensão.

O projeto "Sarminina Cientistas" foi proposto no sentido de promover ações que visam, não somente fomentar a igualdade de gênero, mas também promover o contato precoce das alunas do ensino básico de escolas públicas da Cidade de São Luís do Maranhão com as áreas de STEM, esclarecendo o papel e os aspectos positivos dessas carreiras, visando o aumento no ingresso de mulheres em áreas predominantemente masculinas.

Observa-se que é de fundamental importância oportunizar a essas meninas meios para que elas adquiram conhecimentos teóricos e práticos sobre a ciência, dando-as condições de entender fenômenos que ocorrem no seu cotidiano, além de contribuir para o engajamento e fixação desses talentos nas áreas de atuação correlatas, tornando-as, no futuro, agentes no desenvolvimento científico e tecnológico, em especial no Maranhão (MA), onde as desigualdades são elevadas e os índices de inclusão social estão aquém do que se possa sonhar para o mínimo de uma adequada qualidade de vida.

Neste trabalho, adotou-se como metodologia uma abordagem dinâmica, composta na apresentação de experimentos interativos, minicursos, oficinas, palestras e apresentação de filmes ressaltando a presença feminina na área de STEM. O público-alvo das ações foi constituído por meninas com idade entre 15 e 17 anos, abrangendo alunas do primeiro e segundo ano do ensino médio.

\section{METODOLOGIA}

O projeto foi executado em três escolas da rede pública da Cidade de São Luís - Maranhão sendo duas a nível estadual e uma a nível federal: Colégio Militar do Corpo de Bombeiros (Colégio 2 de Julho), Liceu Maranhense e Colégio Universitário (COLUN), respectivamente. 
A equipe de execução, formada por professores de diversas áreas da ciência, tecnologia, engenharia e matemática, contou com a colaboração de um grupo de alunas de graduação (bolsistas ou voluntárias) que acompanhavam os professores, ajudando-os no desenvolvimento das atividades.

Desde o início do ano letivo de 2019, o diálogo com as três escolas, para planejamento e organização das ações, passou a ser constante para o grupo de professores do Projeto Sarminina Cientistas. Em cada escola, contávamos com o acompanhamento de uma professora (coordenadora local junto à equipe), com o papel de manter as alunas engajadas com as atividades a serem realizadas. Complementarmente, cada escola possuía bolsistas de uma determinada área do projeto - por exemplo Robótica, Matemática, etc. - sendo que as bolsistas aprendiam sobre essa área com os professores e alunos da universidade e poderiam atuar como agente multiplicador desse conhecimento na sua escola. As bolsistas eram estimuladas a participar das atividades de mais de uma área, ou seja, a bolsista de Robótica poderia também participar das atividades de Matemática e vice-versa.

As atividades efetivadas ao longo do ano de 2019, dotadas das mais diferentes metodologias de sensibilização e formação foram: (i) apresentação do projeto as alunas e bate papo com professoras e alunas das áreas de STEM; (ii) mostra de filmes; (iii) atividade de introdução à robótica e (iv) oficinas. Nesta seção serão apresentados os aspectos metodológicos adotados para a realização dessas atividades. É importante destacar, que na metodologia utilizada, cada membro da equipe ficou responsável pela condução de pelo menos uma das atividades.

\subsection{Apresentação do projeto e bate papo com professoras e alunas das áreas de} STEM

Inicialmente, em cada escola participante, foi proferida uma palestra de apresentação do projeto, seguida de um bate papo com professoras e alunas de graduação das áreas de STEM.

O objetivo do bate papo foi o de proporcionar o contato das alunas com mulheres que atuam na área de STEM, desmistificando a ideia de que esta área não é adequada às mulheres, além de transmitir relatos de experiência de alunas de graduação, contando suas trajetórias ensino médio/universidade e vivência em um curso superior, predominantemente masculino.

A Figura 1 registra a ocasião da palestra de apresentação do projeto em uma das escolas.

Figura 1: Palestra de apresentação do projeto (a) bate papo com professoras (b) relato de experiência de alunas de graduação

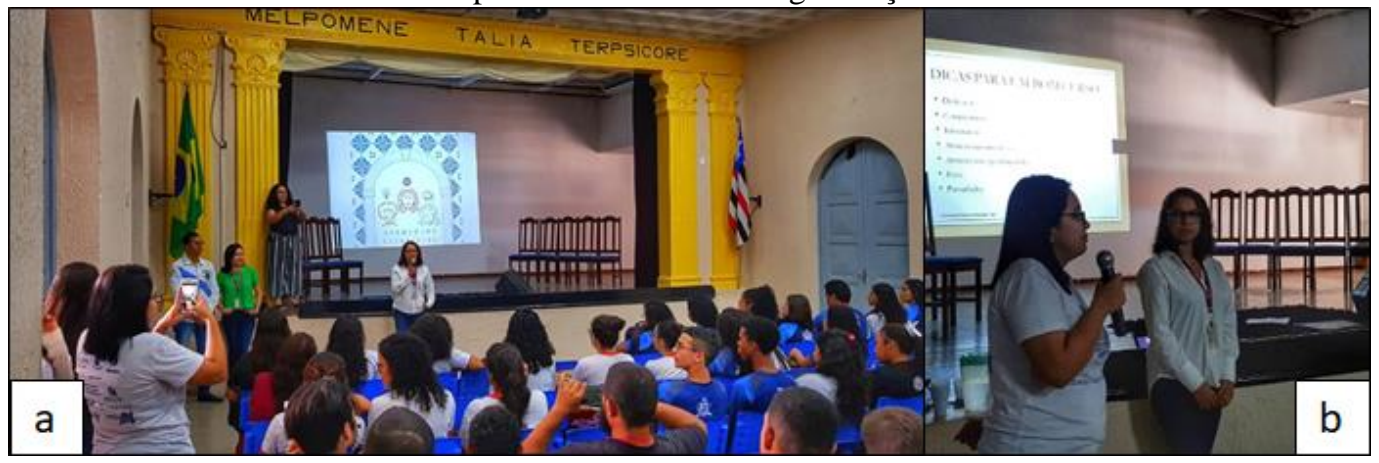

Fonte: Arquivo pessoal da equipe do projeto (2019) 
Adicionalmente, além do feminino (público alvo), a palestra foi aberta aos demais alunos, que foi altamente relevante para que este público entendesse o cenário (problemas, preconceitos, entre outros) para as mulheres nas áreas de STEM.

\subsection{Mostra de filmes}

Em outro momento, foi realizado o "Cinema com Ciência", com a exibição do filme "Estrelas Além do Tempo", que ressalta a história real de três cientistas negras que trabalharam na NASA durante a década de 1960 e colaboraram para a conquista espacial. Após a sessão do filme foi realizado um debate, cuja discussão versou sobre a invisibilidade da mulher na área tecnológica e a equidade de gênero, uma das temáticas abordadas no filme.

A Figura 2 mostra uma das sessões do filme.

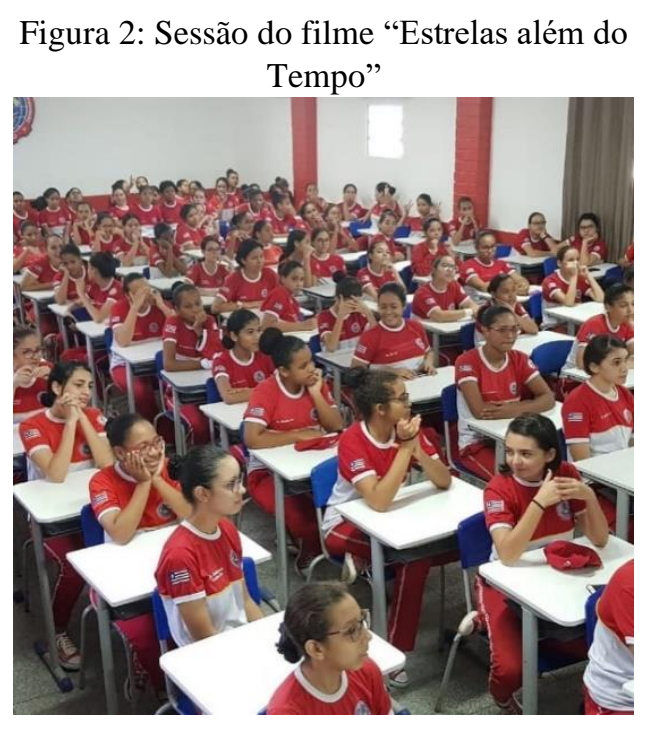

Fonte: Arquivo pessoal da equipe do projeto (2019)

\subsection{Atividade de Introdução à Robótica: Programação com Kits de Robótica}

As atividades de robótica foram realizadas com as bolsistas de cada escola. Cada uma das três escolas tinha uma bolsista para a área de robótica. Entretanto, como uma bolsista de uma área também poderia participar das atividades de outras áreas, na atividade de robótica geralmente tinham-se 3 bolsistas.

Cada escola recebeu um kit com diversos componentes eletrônicos, tais como resistores, diodos, LEDs, sensores, atuadores, assim como Arduinos (Uno e Mega). Adicionalmente, ferramentas - tais como multímetro digital e um kit jogo de chaves - também foram entregues as escolas, assim como caixa organizadora, kit chassi 2WD e kit chassi 4WD. Complementarmente, um kit carregador com baterias/pilhas recarregáveis.

Cada escola recebeu seu kit em um dia diferente, sendo que nesse dia ministrou-se um curso sobre Arduino abordando temas como eletrônica, sinais analógicos/digitais, pinos de entrada e saída, introdução a programação $(\mathrm{C} / \mathrm{C}++)$, etc.

A Figura 3 apresenta fotos capturadas durante a realização desta atividade. 
Figura 3: As alunas durante a atividade de robótica: (a) montagem de circuitos eletrônicos (b) montagem do robô

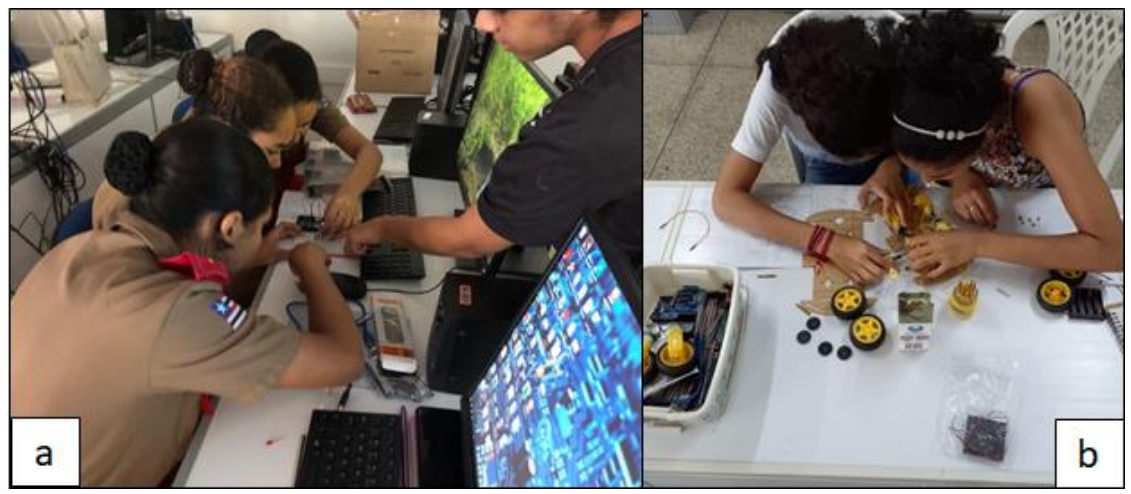

Fonte: Arquivo pessoal da equipe do projeto (2019)

Para a realização das atividades de introdução à robótica, a metodologia foi estruturada em três momentos. No primeiro momento, eram apresentados os conceitos de matemática e física relacionados ao conteúdo do experimento da atividade. No segundo momento, as alunas realizavam as montagens dos circuitos e a programação do microcontrolador. No último momento integravam o circuito eletrônico, programação e montagem do robô. Todas as atividades foram supervisionadas pelo professor responsável pela ação com a colaboração de uma bolsista de graduação do projeto.

\section{$2.4 \quad$ Oficinas}

\section{Jogos Educacionais: Introdução a Lógica de Programação e Desenvolvimento de Jogos}

O ensino de pensamento computacional também foi trabalhado no projeto. A estratégia adotada para estimular as meninas no contato com a informática constituiu na apresentação e utilização da plataforma Scracth, desenvolvida pelo Massachusetts Institute of Technology (MIT) e disponível gratuitamente no site do Scratch Brasil ${ }^{2}$. Através do emprego de uma abstração com peças de quebra-cabeças e usando uma interface gráfica, as meninas deram os primeiros passos na programação de computadores.

A Figura 4 mostra momento registrado durante a realização desta atividade.

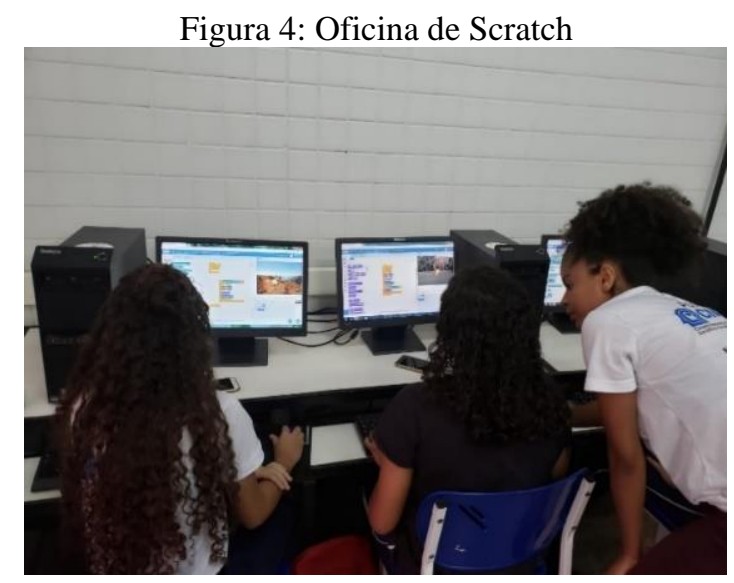

Fonte: Arquivo pessoal da equipe do projeto (2019)

${ }^{2}$ http://www.scratchbrasil.net.br/ 
A oficina foi ministrada em forma de tutorial da ferramenta, contemplando atividades para o raciocínio lógico e dinâmicas para trabalhar conceitos iniciais de algoritmos e programação. Por meio de atividades lúdicas foi trabalhado o tripé de abstrações, decomposições e identificação de padrões que norteiam o ensino do pensamento computacional.

Em seguida, foi apresentada uma breve introdução aos feitos de mulheres que fizeram história na tecnologia. A intenção foi provocar as participantes da oficina para criarem conteúdo usando o Scratch sobre esse tema.

Por fim, as participantes receberam tutoria remota por três semanas em uma dinâmica que as alunas puderam pôr em prática os conhecimentos adquiridos no desenvolvimento de um mini jogo ou uma apresentação interativa sobre importantes cientistas da computação.

Essa ação foi desenvolvida por meninas para meninas. Segundo Pinto et al (2020), ações desenvolvidas por meninas para meninas, é uma forma de mostrar que caso elas decidam seguir nessa carreira, terão o apoio de mulheres dentro do curso.

\section{Oficina de introdução ao MATLAB}

Como estratégia para facilitar o processo de ensino-aprendizagem em matemática básica, foi utilizado o software MATLAB, que é um software computacional conhecido mundialmente como uma excelente ferramenta para soluções de problemas matemáticos e de engenharia, possuindo comandos de fácil interpretação e manipulação.

A Figura 5 registrada momento de realização desta atividade.

Figura 5: Oficina de MATLAB

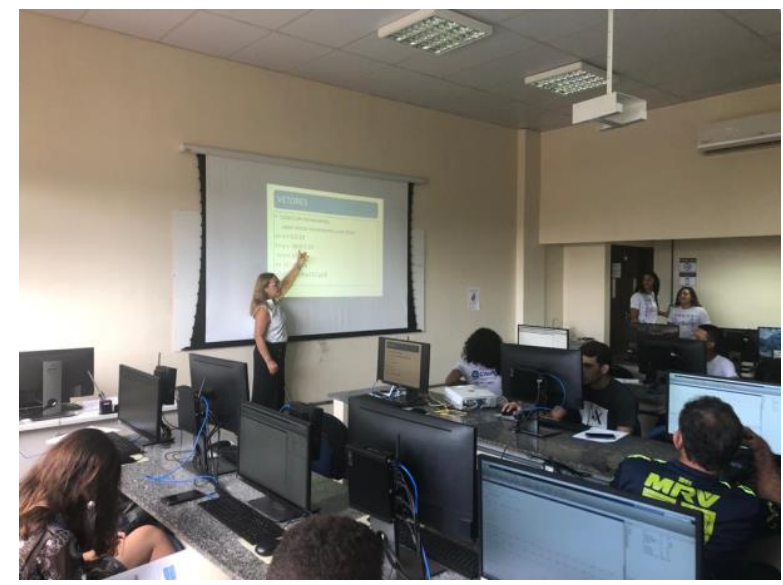

Fonte: Arquivo pessoal da equipe do projeto (2019)

A metodologia utilizada foi estruturada em dois momentos. Primeiramente, foi exposta a teoria, composta dos seguintes conteúdos: fundamentos do MATLAB, operações básicas, funções matemáticas elementares: adição, subtração, multiplicação e divisão; funções matemáticas específicas: matrizes e vetores, e em seguida realizados exemplos práticos aplicando o conteúdo.

\section{Oficina de introdução ao SCILAB}

Nesta oficina a proposta foi da utilização do software livre SCILAB, um ambiente gráfico desenvolvido para cálculo científico, desenvolvido por pesquisadores do Institut Nationale de Recherche en Informatique et en Automatique (INRIA) e da Ecole Nationale des Ponts et Chaussee (ENPC), como uma ferramenta de apoio ao processo de ensino-aprendizagem em estatística. 
A oficina teve como objetivo introduzir conceitos fundamentais de estatística e resumo das medidas de posição e medidas de dispersão com o uso do SCILAB.

A Figura 6 mostra as alunas ao final da oficina.

Figura 6: Oficina de SCILAB

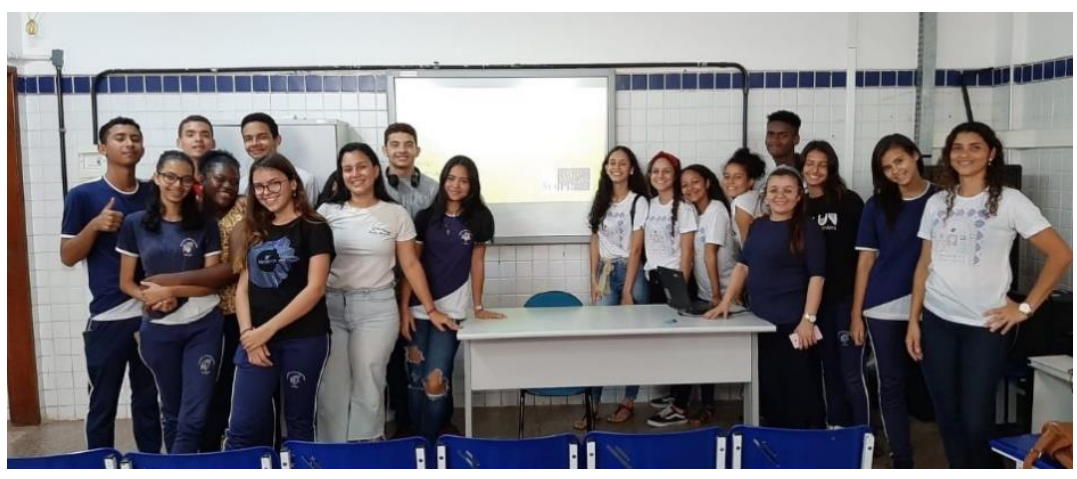

Fonte: Arquivo pessoal da equipe do projeto (2019)

A metodologia utilizada consistiu em apresentar os principais comandos e operações no Scilab para calcular as medidas de posição: moda, média, mediana, percentis e as medidas de dispersão: amplitude, intervalo interquartil, variância, desvio padrão e coeficiente de variância, seguido da realização de exemplos práticos.

Ao final das atividades, foi aplicado um questionário eletrônico ${ }^{3}$ para avaliar a percepção das alunas participantes sobre as ações realizadas e o que poderia ser melhorado em ações futuras.

\section{RESULTADOS E DISCUSSÕES}

A percepção das alunas, em relação as ações implementadas, foram analisadas de forma quantitativa através de questionário. Os resultados obtidos são apresentados nas Figuras 7, 8 e 9.

Figura 7: (a) Grau de satisfação em relação as ações do Projeto (b) Contribuição do projeto para uma melhor percepção das áreas de STEM
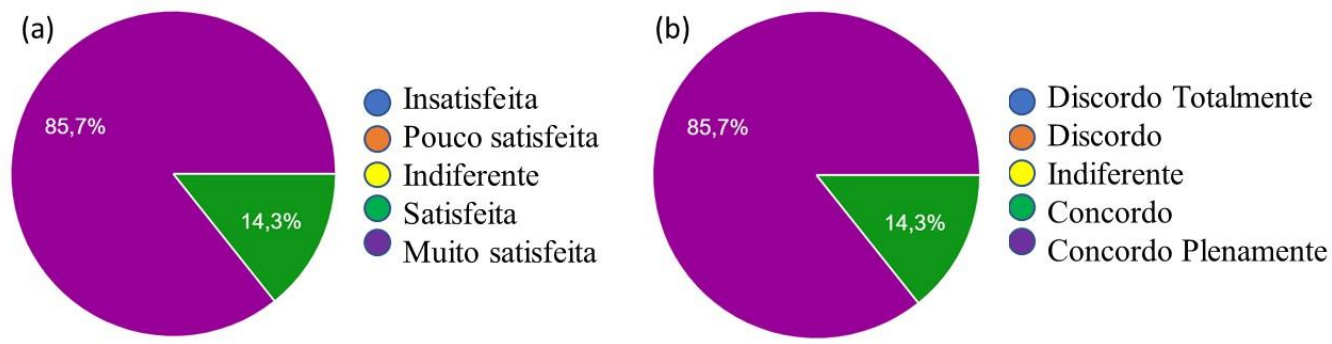

Fonte: Questionário próprio elaborado pela equipe da ação

\footnotetext{
${ }^{3}$ https://forms.gle/2deMvKzbAV3nd7116
} 
A partir da análise da Figura 7a podemos observar que o grau de satisfação com relação as ações do projeto é de $100 \%$.

Quando questionadas se as ações do projeto ajudaram a perceber a área de STEM de uma forma diferente (Figura $7 \mathrm{~b}$ ), $86 \%$ das entrevistadas afirmaram que concordavam plenamente, o que foi percebido durante o desenvolvimento das ações, à exemplo das oficinas. Todas apontaram que as oficinas foram bastante didáticas, interessantes e que possibilitou um maior conhecimento das áreas de STEM. As meninas puderam vivenciar fenômenos vistos teoricamente na escola, instigando então sua curiosidade e percepção.

Figura 8: (a) Interesse das alunas na área de STEM após participação no projeto; b) Percentual de alunas que considerariam seguir carreira em STEM após participação nas ações do projeto

(a)

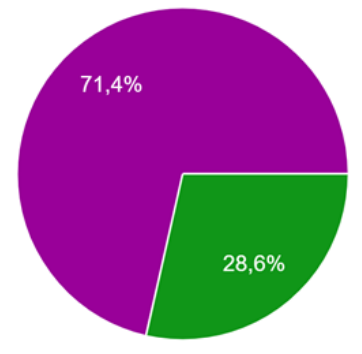

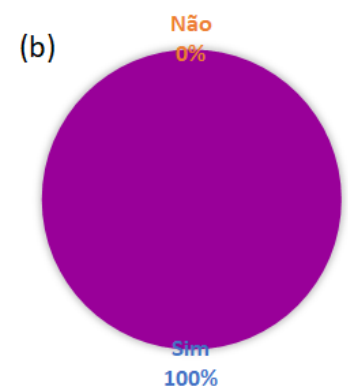

Fonte: Questionário próprio elaborado pela equipe da ação

A partir da análise da Figura 8a concluímos que as alunas de ensino médio em sua grande maioria, $71 \%$ concordaram plenamente que a sua participação nas ações do projeto deixou-as mais interessada na área de STEM. Quando questionadas se seguirão uma carreira na área STEM, 100\% afirmaram que sim. Dos cursos elencados pelas alunas para seguirem na área, podemos citar: engenharia da computação; engenharia química; engenharia civil e engenharia elétrica.

Obtivemos diversos relatos positivos por parte das alunas, de modo que percebemos que existe o interesse do público feminino pela área quando os conteúdos abordados em sala de aula são apresentados na forma lúdica e relacionados com a prática.

Figura 9: (a) Desempenho nos estudos após participação no projeto; b) Implementações de ações nas escolas

(a)

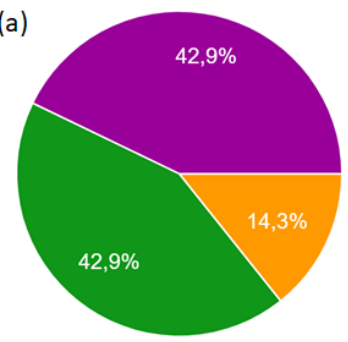

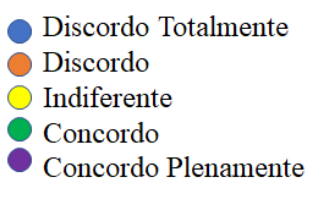

(b)

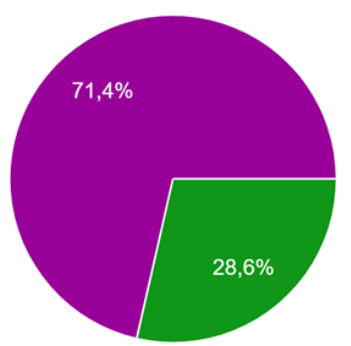

Fonte: Questionário próprio elaborado pela equipe da ação

A Figura 9a demostra o impacto obtido na vida acadêmica das alunas. A maioria das alunas $(86 \%)$ afirmaram que as ações do projeto fizeram melhorar no desempenho nos estudos. 
Por fim, na Figura 9b, podemos observar que a maioria absoluta das entrevistadas afirmaram que a implementação de ações nas escolas, semelhantes ao Projeto Sarminina Cientistas, poderiam contribuir para que um maior público de meninas se interesse pela área de STEM.

Os resultados apresentados neste trabalho estão em acordo com dados publicados em ações realizadas anteriormente pela equipe do projeto. Estes, apontam que a implementação de atividades ligadas as áreas de STEM contribuiu para um maior interesse das alunas pelas áreas de ciência, tecnologia e/ou engenharia (DE LA SALLES et al, 2019).

\section{CONSIDERAÇÕES FINAIS}

O projeto Sarminina Cientistas foi desenvolvido para despertar o interesse de alunas do ensino médio de escolas públicas por carreiras em STEM, através de atividades conduzidas por professores e/ou estudantes de graduação destas áreas.

As atividades desenvolvidas com as alunas das escolas públicas foram de grande importância, pois demonstraram a necessidade de se implantar alternativas de aprendizagem que estimulem a curiosidade das meninas para as áreas de STEM. Acredita-se que essas ações possam contribuir para desmistificar a imagem de que esta área não é adequada para o público feminino, contribuindo com a quebra de preconceitos e abrindo as suas mentes para profissões até então não cogitadas, revelando a importância do projeto como meio para despertar o interesse destas alunas pela área tecnológica.

Espera-se que a partir desse relato de experiência, seja possível a aplicação de mais projetos com finalidades similares, para que o engajamento de alunas de ensino médio à área de STEM seja cada vez maior.

\section{Agradecimentos}

A equipe envolvida neste trabalho agradece ao Conselho Nacional de Desenvolvimento Científico e Tecnológico - $\mathrm{CNPq}$ pelo financiamento do projeto (processo CNPq $\mathrm{n}^{\circ}$ 442951/2018-3), bem como, as escolas públicas participantes das ações.

\section{REFERÊNCIAS}

BOLZANI, V. da S. Mulheres na ciência: por que ainda somos tão poucas? Ciência e Cultura. São Paulo, v. 69, n. 4, p. 56-59, 2017.

DE LA SALLES, Kátia. S. T; DE LA SALLES, Wendell F.; FERREIRA, Maira S.; SOUSA, Janyeid K. C., RIBEIRO, Paulo R.A. Despertando Vocações para as Carreiras de Exatas e Tecnologia: O Projeto Sarminina Cientistas. In: XLVII Congresso Brasileiro de Educação em Engenharia, 2019, Ceará. Anais. Fortaleza, 2019.

NORTE, B. D. Mulheres nas Exatas. Disponível em: https://revistaensinosuperior.com.br/mulheres-nas-exatas/. Acesso em: 1 de maio. 2020.

PINTO, Nathasha; ARAUJO, Alana; MENDES, Estephane; PAVANI, Giovanna; SILVA, Karla; NETO, Raimundo; SALLES, Katia; RIVERO, Luis. Quando a Aluna se Torna a Mestre: Um Relato da Experiência de Alunas de Graduação Aplicando Dinâmicas de Ensino de Computação para Alunas de Ensino Médio. In: WORKSHOP SOBRE EDUCAÇÃO EM COMPUTAÇÃO (WEI), 28., 2020, Cuiabá. Anais [...]. Porto Alegre: Sociedade Brasileira de Computação, 2020. p. 36-40. ISSN 2595-6175. DOI: https://doi.org/10.5753/wei.2020.11125. 
SIRIMANNE, S. N. What if our future were designed equally by women? Disponível em https://unctad.org/en/pages/newsdetails.aspx? OriginalVersionID=1993 Acesso em: 1 de maio. 2020.

UNITED NATIONS EDUCATIONAL, SCIENTIFIC AND CULTURAL ORGANIZATION. Gender and Education for All: The Leap to Equality. Place de Fontenoy, 75352 Paris 07 SP. 2003. ISBN 92-3-103914-8.

\title{
ENCOURAGING GIRLS IN THE MARANHÃO STATE IN STEM RELATED CAREERS
}

\begin{abstract}
In order to demystify the area of Science, Technology, Engineering and Mathematics (STEM), as well as to increase the interest of girls to pursue careers in these areas, a group of professors in the areas of science, technology and engineering at the Federal University of Maranhão proposed the project "Sarminina Scientists: Stimulating Girls from Maranhão for Technology Careers". The project adopted a dynamic approach as methodology - that is, playful and active learning activities - aiming at the appeal, encouragement and curiosity of students from public schools in São Luís city (Maranhão state) for the STEM areas. Of the various actions carried out, we can mention the activities in the area of robotics; workshops on the development of computational thinking and the introduction to SCILAB and MATLAB; film shows, as well as professional orientation lectures. All actions were planned in order to provide students with an approach to an area that is often undermined by a large part of the students, mainly by the female audience. Throughout the actions, we noticed several positive impacts, so that we could conclude that the activities helped the students to perceive the STEM area in a different way, correlating the subjects covered with the subjects of mathematics, physics, chemistry and computing.
\end{abstract}

Keywords: Girls. STEM. Public School. Sarminina Cientistas. 